MATHEMATICS OF COMPUTATION

Volume 74, Number 251, Pages 1201-1216

S 0025-5718(05)01751-5

Article electronically published on February 16, 2005

\title{
OPTIMAL ERROR ESTIMATE \\ OF THE PENALTY FINITE ELEMENT METHOD \\ FOR THE TIME-DEPENDENT \\ NAVIER-STOKES EQUATIONS
}

\author{
YINNIAN HE
}

\begin{abstract}
A fully discrete penalty finite element method is presented for the two-dimensional time-dependent Navier-Stokes equations. The time discretization of the penalty Navier-Stokes equations is based on the backward Euler scheme; the spatial discretization of the time discretized penalty Navier-Stokes equations is based on a finite element space pair $\left(X_{h}, M_{h}\right)$ which satisfies some approximate assumption. An optimal error estimate of the numerical velocity and pressure is provided for the fully discrete penalty finite element method when the parameters $\epsilon, \Delta t$ and $h$ are sufficiently small.
\end{abstract}

\section{INTRODUCTION}

In this article, we consider the time-dependent Navier-Stokes equations

$$
\begin{aligned}
u_{t} & -\nu \Delta u+(u \cdot \nabla) u+\nabla p=f, \operatorname{div} u=0,(x, t) \in \Omega \times(0, T], \\
u & =0,(x, t) \in \partial \Omega \times(0, T], u(x, 0)=u_{0}(x), x \in \Omega,
\end{aligned}
$$

where $\Omega$ is an open bounded set in $R^{2}$ with a smooth boundary $\partial \Omega$ being of class $C^{2}$, or $\Omega$ is a plane convex polygon, $u=u(x, t)=\left(u_{1}(x, t), u_{2}(x, t)\right)$ represents the velocity vector of a viscous incompressible fluid, $p=p(x, t)$ the pressure, $f=f(x, t)$ the prescribed body force, $u_{0}(x)$ the initial velocity, $\nu>0$ the viscosity, and $T>0$ a finite time.

We note that the velocity $u$ and the pressure $p$ in (1.1)-(1.2) are coupled together by the incompressibility constraint "div $u=0$ ", which makes the system difficult to solve numerically. A popular strategy to overcome this difficulty is to relax the incompressibility constraint in an appropriate way, resulting in a class of pseudo-compressibility methods, among which are the penalty method, the artificial compressibility method, the pressure stabilization method and the projection method (see for instance [3, 4, 5, 7, 8, 9, 12, 14, 16, 17, 18, 19, 20, 21]).

Received by the editor July 2, 2003 and, in revised form, May 15, 2004.

2000 Mathematics Subject Classification. Primary 35L70, 65N30, 76D06.

Key words and phrases. Navier-Stokes problem, penalty finite element method, backward Euler scheme, error estimate.

This work was subsidized by the Special Funds for Major State Basic Research Projects G1999032801-07, NSF of China 10371095. 
The penalty method applied to (1.1)-(1.2) is to approximate the solution $(u, p)$ by $\left(u_{\epsilon}, p_{\epsilon}\right)$ satisfying the following penalty Navier-Stokes equations:

$$
\begin{aligned}
& u_{\epsilon t}-\nu \Delta u_{\epsilon}+B\left(u_{\epsilon}, u_{\epsilon}\right)+\nabla p_{\epsilon}=f, \operatorname{div} u_{\epsilon}+\frac{\epsilon}{\nu} p_{\epsilon}=0,(x, t) \in \Omega \times(0, T], \\
& u_{\epsilon}=0,(x, t) \in \partial \Omega \times(0, T], u_{\epsilon}(x, 0)=u_{0}(x), x \in \Omega,
\end{aligned}
$$

where $B(u, v)=(u \cdot \nabla) v+\frac{1}{2}(\operatorname{div} u) v$ is the modified bilinear term, introduced by Temam [19] to ensure the dissipativity of equations (1.3)-(1.4). We note also that $p_{\epsilon}$ in (1.3)-(1.4) can be eliminated to obtain a penalty system of $u_{\epsilon}$ only, which is much easier to solve than the original equations (1.1)-(1.2). Hence the penalty method has been widely used in many areas of computational fluid dynamics (see for instance [1, 13]). It is well known [19] that $\lim _{\epsilon \rightarrow 0}\left(u_{\epsilon}(t), p_{\epsilon}(t)\right)=(u(t), p(t))$, the solution of (1.1)-(1.2). It has also been known [2] that the attractors generated by the penalty equations (1.3)-(1.4) converge to the attractor of the Navier-Stokes equations (1.1)(1.2). The error bound of $\left(u_{\epsilon}, p_{\epsilon}\right)$ to $(u, p)$, to the author's knowledge, has been provided by Shen [17] and Huang and Li [12] and the error bound is

$$
\sup _{0 \leq t \leq T}\left\|u(t)-u_{\epsilon}(t)\right\|_{L^{2}}+\left(\int_{0}^{T}\left(\left\|u-u_{\epsilon}\right\|_{H^{1}}^{2}+\left\|p-p_{\epsilon}\right\|_{L^{2}}^{2}\right) d t\right)^{1 / 2} \leq \kappa \epsilon^{1 / 2},
$$

where $\kappa>0$ is a general positive constant depending on the data $\left(\nu, u_{0}, f, \Omega, T\right)$, which may stand for different values at its different occurrences.

However, the best error estimate available, to the author's knowledge, is

$$
\begin{aligned}
\sup _{0 \leq t \leq T}\left(\tau^{1 / 2}(t)\left\|u(t)-u_{\epsilon h}(t)\right\|_{L^{2}}\right. & \left.+\tau(t)\left\|u(t)-u_{\epsilon h}(t)\right\|_{H^{1}}\right) \\
& +\left(\int_{0}^{T} \tau^{2}(t)\left\|p-p_{\epsilon}\right\|_{L^{2}}^{2} d t\right)^{1 / 2} \leq \kappa \epsilon,
\end{aligned}
$$

where $\tau(t)=\min \{t, 1\}$; the reader can refer to Shen [16] for the detail. Furthermore, when the backward Euler scheme is applied to the penalty Navier-Stokes equations (1.3)-(1.4), Shen [16] has provided the following optimal error estimate:

$$
\begin{aligned}
\sup _{1 \leq n \leq N}\left(\tau^{1 / 2}\left(t_{n}\right)\left\|u\left(t_{n}\right)-u_{\epsilon}^{n}\right\|_{L^{2}}\right. & \left.+\tau\left(t_{n}\right)\left\|u\left(t_{n}\right)-u_{\epsilon}^{n}\right\|_{H^{1}}\right) \\
& +\left(\Delta t \sum_{n=1}^{N} \tau^{2}\left(t_{n}\right)\left\|p\left(t_{n}\right)-p_{\epsilon}^{n}\right\|_{L^{2}}^{2}\right)^{1 / 2} \leq \kappa(\epsilon+\Delta t),
\end{aligned}
$$

where $0<\Delta t<1$ is the time-step size, $t_{n}=n \Delta t, t_{N}=T,\left(u_{\epsilon}^{n}, p_{\epsilon}^{n}\right)$ is an approximation of $(u, p)$ at time $t_{n}$.

In this paper, we aim to extend the work of Shen 16 to the case of a fully discrete penalty finite element method for the time-dependent Navier-Stokes equations and provide the optimal error estimates for the penalty finite element solution $\left(u_{\epsilon h}^{n}, p_{\epsilon h}^{n}\right)$. Under the assumption $\left(A_{1}\right)$ about the data $\left(u_{0}, f\right)$ and the assumption $\left(A_{2}\right)$ about the finite element space pair $\left(X_{h}, M_{h}\right)$, we provide the following optimal error estimate:

$$
\begin{aligned}
\sup _{1 \leq n \leq N} \tau\left(t_{n}\right)\left\|u\left(t_{n}\right)-u_{\epsilon h}^{n}\right\|_{H^{1}} & +\left(\Delta t \sum_{n=1}^{N} \tau^{2}\left(t_{n}\right)\left\|p\left(t_{n}\right)-p_{\epsilon h}^{n}\right\|_{L^{2}}^{2}\right)^{1 / 2} \\
\leq & \kappa(\epsilon+h+\Delta t),
\end{aligned}
$$

for sufficient small $\epsilon, \Delta t$ and $h$. 
The remainder of the paper is organized as follows. In the next section, we introduce some notation and preliminary results for the time-dependent penalty Navier-Stokes equations (1.3)-(1.4). In $\S 3$, we provide some regularity results for the time discretized penalty Navier-Stokes equations with the Euler backward scheme. The fully discrete finite element method of the penalty Navier-Stokes equations (1.3)-(1.4) is presented in $\S 4$ and some boundedness results of the numerical solution $\left(u_{\epsilon h}^{n}, p_{\epsilon h}^{n}\right), 1 \leq n \leq N$, are provided in this section. The optimal error estimate is obtained for the fully discrete penalty finite element method in $\S 5$.

\section{Preliminaries}

In this section, we aim to describe some of the notation and results which will be frequently used in this paper. For the mathematical setting of the NavierStokes equations (1.1)-(1.2) and the penalty Navier-Stokes equations (1.3)-(1.4), we introduce the Hilbert spaces

$$
X=H_{0}^{1}(\Omega)^{2}, Y=L^{2}(\Omega)^{2}, M=\left\{q \in L^{2}(\Omega) ; \int_{\Omega} q d x=0\right\} .
$$

The spaces $L^{2}(\Omega)^{m}, m=1,2,4$, are endowed with the $L^{2}$-scalar product and $L^{2}$ norm denoted by $(\cdot, \cdot)$ and $\|\cdot\|_{0}$, respectively. The space $X$ is equipped with the usual scalar product $(\nabla u, \nabla v)$ and norm $\|\nabla u\|_{0}$.

We define $A u=-\Delta u$ and $A_{\epsilon} u=-\Delta u-\frac{1}{\epsilon} \nabla \operatorname{div} u$, which are the operators associated with the Navier-Stokes equations and the penalty Navier-Stokes equations. They are the positive self-adjoint operators from $D(A)=H^{2}(\Omega)^{2} \cap X$ onto $Y$ and the powers $A^{\alpha}$ and $A_{\epsilon}^{\alpha}$ of $A$ and $A_{\epsilon}(\alpha \in R)$ are well defined. In particular,

$$
\left(A^{1 / 2} u, A^{1 / 2} v\right)=(\nabla u, \nabla v),\left(A_{\epsilon}^{1 / 2} u, A_{\epsilon}^{1 / 2} v\right)=\left(A^{1 / 2} u, A^{1 / 2} v\right)+\frac{1}{\epsilon}(\operatorname{div} u, \operatorname{div} v)
$$

hold for all $u, v \in X$.

It is well known that the following Gagliardo-Nirenberg inequalities hold:

$$
\begin{aligned}
\|v\|_{L^{4}} & \leq c\|v\|_{0}^{1 / 2}\left\|A^{1 / 2} v\right\|_{0}^{1 / 2},\|v\|_{0} \leq c\left\|A^{1 / 2} v\right\|_{0}, \forall v \in X \\
\|\nabla v\|_{L^{4}} & \leq c\left\|A^{1 / 2} v\right\|_{0}^{1 / 2}\|A v\|_{0}^{1 / 2},\left\|A^{1 / 2} v\right\|_{0} \leq c\|A v\|_{0}, \forall v \in D(A), \\
\|v\|_{L^{\infty}} & \leq c\|v\|_{0}^{1 / 2}\|A v\|_{0}^{1 / 2}, \forall v \in D(A)
\end{aligned}
$$

where $c$ is a general positive constant depending only on $\Omega$, which may stand for different values at its different occurrences.

Furthermore, we recall the following lemma given in [2, 16].

Lemma 2.1. There exists a constant $c_{0}>0$ depending only on $\Omega$ and such that if $\epsilon c_{0} \leq 1$,

$$
\|A v\|_{0} \leq c_{0}\left\|A_{\epsilon} v\right\|_{0},\left\|A^{1 / 2} v\right\|_{0} \leq c_{0}\left\|A_{\epsilon}^{1 / 2} v\right\|_{0} .
$$

As for the time-dependent Navier-Stokes equations (1.1)-(1.2) and the timedependent penalty Navier-Stokes equations (1.3)-(1.4), we define the continuous bilinear forms

$$
\begin{gathered}
(u, v)=\nu\left(A^{1 / 2} u, A^{1 / 2} v\right), a_{\epsilon}(u, v)=\nu\left(A_{\epsilon}^{1 / 2} u, A_{\epsilon}^{1 / 2} v\right), \forall u, v \in X, \\
d(v, q)=(\operatorname{div} v, q), \forall v \in X, q \in M,
\end{gathered}
$$


respectively. We also introduce a continuous trilinear form on $X \times X \times X$

$$
\begin{array}{r}
b(u, v, w)=\langle B(u, v), w\rangle_{X^{\prime}, X}=((u \cdot \nabla) v, w)+\frac{1}{2}((\operatorname{div} u) v, w) \\
=\frac{1}{2}((u \cdot \nabla) v, w)-\frac{1}{2}((u \cdot \nabla) w, v) \forall u, v, w \in X .
\end{array}
$$

It is easy to verify that $b$ satisfies the following important property:

$$
b(u, v, w)=-b(u, w, v), \forall u, v, w \in X .
$$

We usually make the following assumption on the prescribed data $\left(u_{0}, f\right)$ :

$\left(A_{1}\right)$ The initial velocity $u_{0}(x) \in D(A)$ with $\operatorname{div} u_{0}=0$ and the forcing function $f(x, t) \in H^{1, \infty}(0, T ; Y)$ satisfy

$$
\left\|A u_{0}\right\|_{0}+\sup _{t \in[0, T]}\left\{\|f(t)\|_{0}+\left\|f_{t}(t)\right\|_{0}\right\} \leq C,
$$

for some positive constant $C$.

With the above notation, the Navier-Stokes formulation related to (1.1)-(1.2) and the penalty Navier-Stokes formulation related to (1.3)-(1.4) are defined, respectively, as follows: find $(u, p) \in L^{\infty}(0, T ; Y) \cap L^{2}(0, T ; X) \times L^{2}(0, T ; M)$ such that

$$
\left(u_{t}, v\right)+a(u, v)-d(v, p)+d(u, q)+b(u, u, v)=(f, v), \forall(v, q) \in(X, M),
$$

and find $\left(u_{\epsilon}, p_{\epsilon}\right) \in L^{\infty}(0, T ; Y) \cap L^{2}(0, T ; X) \times L^{2}(0, T ; M)$ such that for all $(v, q) \in$ $X \times M$

$$
\left(u_{\epsilon t}, v\right)+a\left(u_{\epsilon}, v\right)-d\left(v, p_{\epsilon}\right)+d\left(u_{\epsilon}, q\right)+\frac{\epsilon}{\nu}\left(p_{\epsilon}, q\right)+b\left(u_{\epsilon}, u_{\epsilon}, v\right)=(f, v),
$$

with the initial conditions $u(0)=u_{0}$ and $u_{\epsilon}(0)=u_{0}$, respectively.

Now, let us consider the time discretization of the penalized Navier-Stokes formulation (2.7) by the backward Euler scheme

$$
\left(d_{t} u_{\epsilon}^{n}, v\right)+a\left(u_{\epsilon}^{n}, v\right)-d\left(v, p_{\epsilon}^{n}\right)+d\left(u_{\epsilon}^{n}, q\right)+\frac{\epsilon}{\nu}\left(p_{\epsilon}^{n}, q\right)+b\left(u_{\epsilon}^{n}, u_{\epsilon}^{n}, v\right)=\left(f\left(t_{n}\right), v\right),
$$

for all $(v, q) \in X \times M$ and $1 \leq n \leq N$, where $0<\Delta t<1$ is the time-step size, $t_{n}=n \Delta t, t_{N}=T,\left(u_{\epsilon}^{0}, p_{\epsilon}^{0}\right)=\left(u_{0}, 0\right)$ and $d_{t} u_{\epsilon}^{n}=\frac{1}{\Delta t}\left(u_{\epsilon}^{n}-u_{\epsilon}^{n-1}\right)$ for $1 \leq n \leq N$, and $d_{t} u_{\epsilon}^{0}$ is defined to satisfy

$$
\left(d_{t} u_{\epsilon}^{0}, v\right)+a\left(u_{0}, v\right)+\left(\left(u_{0} \cdot \nabla u_{0}\right), v\right)=(f(0), v), \forall v \in X \text { with } \operatorname{div} v=0 .
$$

Hence, by using (2.3), it holds that

$$
\begin{aligned}
\left\|d_{t} u_{\epsilon}^{0}\right\|_{0} & \leq \nu\left\|A u_{0}\right\|_{0}+\left\|\left(u_{0} \cdot \nabla\right) u_{0}\right\|_{0}+\|f(0)\|_{0} \\
& \leq 2 \nu\left\|A u_{0}\right\|_{0}+c\left\|u_{0}\right\|_{0}\left\|A^{1 / 2} u_{0}\right\|_{0}^{2}+\|f(0)\|_{0} .
\end{aligned}
$$

Theorem 2.2. Suppose that $\left(A_{1}\right)$ and $\epsilon c_{0} \leq 1$ are valid. The following error estimate holds:

$$
\tau^{2}\left(t_{m}\right)\left\|A^{1 / 2}\left(u\left(t_{m}\right)-u_{\epsilon}^{m}\right)\right\|_{0}^{2}+\Delta t \sum_{n=1}^{m} \tau^{2}\left(t_{n}\right)\left\|p\left(t_{n}\right)-p_{\epsilon}^{n}\right\|_{0}^{2} \leq \kappa\left(\epsilon^{2}+\Delta t^{2}\right),
$$

for all $1 \leq m \leq N$.

We refer to Shen [16] for the proof of this result.

In this paper, we will frequently use a discrete version of the Gronwall lemmas used in [11, 16]. 
Lemma 2.3. Let $C$ and $a_{n}, b_{n}, d_{n}$, for integers $1 \leq m \leq N$, be nonnegative numbers such that

$$
a_{m}+\Delta t \sum_{n=1}^{m} b_{n} \leq \Delta t \sum_{n=0}^{m-1} a_{n} d_{n}+C, \forall 1 \leq m \leq N .
$$

Assume that $d_{n} \Delta t \leq \frac{1}{2}, \forall 1 \leq n \leq N$. Then

$$
a_{m}+\Delta t \sum_{n=1}^{m} b_{n} \leq C \exp \left(2 \Delta t \sum_{n=1}^{m-1} d_{n}\right), \forall 1 \leq m \leq N .
$$

\section{Regularity}

In order to consider the error bound of the finite element solution related to the penalty Navier-Stokes formulation (2.8), we need the following regularity of functions $\left\{u_{\epsilon}^{n}\right\}_{n=1}^{N}$ and $\left\{p_{\epsilon}^{n}\right\}_{n=1}^{N}$.

Theorem 3.1. Under the assumptions of Theorem 2.2, there is a constant $\kappa_{0}>0$ such that if $\Delta t \kappa_{0} \leq 1$, then

$$
\begin{aligned}
\left\|A_{\epsilon}^{1 / 2} u_{\epsilon}^{m}\right\|_{0}^{2} & +\Delta t \sum_{n=1}^{m}\left(\left\|d_{t} u_{\epsilon}^{n}\right\|_{0}^{2}+\left\|A_{\epsilon} u_{\epsilon}^{n}\right\|_{0}^{2}+\left\|p_{\epsilon}^{n}\right\|_{0}^{2}\right) \leq \kappa, \\
\left\|d_{t} u_{\epsilon}^{m}\right\|_{0}^{2} & +\left\|A_{\epsilon} u_{\epsilon}^{m}\right\|_{0}^{2}+\left\|p_{\epsilon}^{n}\right\|_{1}^{2}+\Delta t \sum_{n=1}^{m}\left\|A_{\epsilon}^{1 / 2} d_{t} u_{\epsilon}^{n}\right\|_{0}^{2} \leq \kappa,
\end{aligned}
$$

for all $1 \leq m \leq N$, where $\|\cdot\|_{1}$ denotes the norm of the Sobolev space $H^{1}(\Omega)$.

Proof. Taking $(v, q)=2\left(u_{\epsilon}^{n}, p_{\epsilon}^{n}\right) \Delta t$ in (2.8), using (2.5) and the relation

$$
2(u-v, u)=\|u\|_{0}^{2}-\|v\|_{0}^{2}+\|u-v\|_{0}^{2}, \forall u, v \in Y,
$$

we have

$$
\left\|u_{\epsilon}^{n}\right\|_{0}^{2}-\left\|u_{\epsilon}^{n-1}\right\|_{0}^{2}+2 \nu\left\|A^{1 / 2} u_{\epsilon}^{n}\right\|_{0}^{2} \Delta t \leq 2\left\|f\left(t_{n}\right)\right\|_{0}\left\|u_{\epsilon}^{n}\right\|_{0} \Delta t .
$$

Summing this inequality from 1 to $m$ and using (2.1) and the Young inequality, we obtain

$$
\left\|u_{\epsilon}^{m}\right\|_{0}^{2}+\nu \Delta t \sum_{n=1}^{m}\left\|A^{1 / 2} u_{\epsilon}^{n}\right\|_{0}^{2} \leq\left\|u_{0}\right\|_{0}^{2}+\nu^{-1} c \Delta t \sum_{n=1}^{N}\left\|f\left(t_{n}\right)\right\|_{0}^{2} \leq \kappa .
$$

Next, we can derive from (2.8) that

$$
d_{t} u_{\epsilon}^{n}+\nu A_{\epsilon} u_{\epsilon}^{n}+B\left(u_{\epsilon}^{n}, u_{\epsilon}^{n}\right)=f\left(t_{n}\right) .
$$

Taking the scalar product of (3.5) with $\left(\nu^{-1} d_{t} u_{\epsilon}^{n}+A_{\epsilon} u_{\epsilon}^{n}\right) \Delta t$ in $Y$ and using the relation

$$
2\left(A_{\epsilon}^{1 / 2}(u-v), A_{\epsilon}^{1 / 2} u\right)=\left\|A_{\epsilon}^{1 / 2} u\right\|_{0}^{2}-\left\|A_{\epsilon}^{1 / 2} v\right\|_{0}^{2}+\left\|A_{\epsilon}^{1 / 2}(u-v)\right\|_{0}^{2}, \forall u, v \in X,
$$

we get

$$
\begin{array}{ll}
\left\|A_{\epsilon}^{1 / 2} u_{\epsilon}^{n}\right\|_{0}^{2} & -\left\|A_{\epsilon}^{1 / 2} u_{\epsilon}^{n-1}\right\|_{0}^{2}+\nu^{-1}\left\|d_{t} u_{\epsilon}^{n}\right\|_{0}^{2} \Delta t+\nu\left\|A_{\epsilon} u_{\epsilon}^{n}\right\|_{0}^{2} \Delta t \\
& +b\left(u_{\epsilon}^{n}, u_{\epsilon}^{n}, \nu^{-1} d_{t} u_{\epsilon}^{n}+A_{\epsilon} u_{\epsilon}^{n}\right) \Delta t=\left(f\left(t_{n}\right), \nu^{-1} d_{t} u_{\epsilon}^{n}+A_{\epsilon} u_{\epsilon}^{n}\right) \Delta t .
\end{array}
$$


By using (2.3)-(2.4), we have

$$
\begin{aligned}
& \left|b\left(u_{\epsilon}^{n}, u_{\epsilon}^{n}, \nu^{-1} d_{t} u_{\epsilon}^{n}+A_{\epsilon} u_{\epsilon}^{n}\right)\right| \\
& \quad \leq c\left\|A^{1 / 2} u_{\epsilon}^{n}\right\|_{0}\left\|u_{\epsilon}^{n}\right\|_{L^{\infty}}\left\|\nu^{-1} d_{t} u_{\epsilon}^{n}+A_{\epsilon} u_{\epsilon}^{n}\right\|_{0} \\
& \quad \leq \frac{1}{4 \nu}\left\|d_{t} u_{\epsilon}^{n}\right\|_{0}^{2}+\frac{\nu}{4}\left\|A_{\epsilon} u_{\epsilon}^{n}\right\|_{0}^{2}+\nu^{-1} c\left\|u_{\epsilon}^{n}\right\|_{0}^{2}\left\|A^{1 / 2} u_{\epsilon}^{n}\right\|_{0}^{2}\left\|A_{\epsilon}^{1 / 2} u_{\epsilon}^{n}\right\|_{0}^{2}, \\
& \quad \leq\left(f\left(t_{n}\right), \nu^{-1} d_{t} u_{\epsilon}^{n}+A_{\epsilon} u_{\epsilon}^{n}\right) \mid \\
& \quad \leq \frac{1}{4 \nu}\left\|d_{t} u_{\epsilon}^{n}\right\|_{0}^{2}+\frac{\nu}{4}\left\|A_{\epsilon} u_{\epsilon}^{n}\right\|_{0}^{2}+\nu^{-1} c\left\|f\left(t_{n}\right)\right\|_{0}^{2} .
\end{aligned}
$$

Combining these estimates with (3.7) yields

$$
\begin{aligned}
\left\|A_{\epsilon}^{1 / 2} u_{\epsilon}^{n}\right\|_{0}^{2} & -\left\|A_{\epsilon}^{1 / 2} u_{\epsilon}^{n-1}\right\|_{0}^{2}+\frac{1}{2}\left(\nu^{-1}\left\|d_{t} u_{\epsilon}^{n}\right\|_{0}^{2}+\nu\left\|A_{\epsilon} u_{\epsilon}^{n}\right\|_{0}^{2}\right) \Delta t \\
& \leq d_{n}\left\|A_{\epsilon}^{1 / 2} u_{\epsilon}^{n}\right\|_{0}^{2} \Delta t+\nu^{-1} c\left\|f\left(t_{n}\right)\right\|_{0}^{2} \Delta t,
\end{aligned}
$$

where $d_{n}=\nu^{-1} c\left(1+\nu^{-2}\left\|u_{\epsilon}^{n}\right\|_{0}^{2}\right)\left\|A^{1 / 2} u_{\epsilon}^{n}\right\|_{0}^{2}$. Summing this inequality from 1 to $m$ and noting $A_{\epsilon}^{1 / 2} u_{\epsilon}^{0}=A^{1 / 2} u_{0}$, we obtain

$$
\begin{aligned}
\left\|A_{\epsilon}^{1 / 2} u_{\epsilon}^{m}\right\|_{0}^{2} & +\frac{1}{2} \Delta t \sum_{n=1}^{m}\left(\nu^{-1}\left\|d_{t} u_{\epsilon}^{n}\right\|_{0}^{2}+\nu\left\|A_{\epsilon} u_{\epsilon}^{n}\right\|_{0}^{2}\right) \\
& \leq\left\|A^{1 / 2} u_{0}\right\|_{0}^{2}+\Delta t \sum_{n=1}^{m} d_{n}\left\|A_{\epsilon}^{1 / 2} u_{\epsilon}^{n}\right\|_{0}^{2}+\nu^{-1} c \Delta t \sum_{n=1}^{N}\left\|f\left(t_{n}\right)\right\|_{0}^{2} \\
& \leq \kappa+\Delta t \sum_{n=1}^{m} d_{n}\left\|A_{\epsilon}^{1 / 2} u_{\epsilon}^{n}\right\|_{0}^{2} .
\end{aligned}
$$

If we choose $\Delta t$ such that $d_{n} \Delta t \leq \frac{1}{2}$, by applying Lemma 2.3 to (3.8), it then holds that

$$
\left\|A_{\epsilon}^{1 / 2} u_{\epsilon}^{m}\right\|_{0}^{2}+\frac{1}{2} \Delta t \sum_{n=1}^{m}\left(\nu^{-1}\left\|d_{t} u_{\epsilon}^{n}\right\|_{0}^{2}+\nu\left\|A_{\epsilon} u_{\epsilon}^{n}\right\|_{0}^{2}\right) \leq k \exp \left(2 \Delta t \sum_{n=1}^{m} d_{n}\right),
$$

for all $1 \leq m \leq N$. From (2.4), (3.4) and (3.9), there exists a constant $\kappa_{0}>0$ such that

$$
2 \Delta t \sum_{n=1}^{m} d_{n} \leq \kappa, d_{m}=\nu^{-1} c\left(1+\nu^{-2}\right)\left\|u_{\epsilon}^{m}\right\|_{0}^{2}\left\|A^{1 / 2} u_{\epsilon}^{m}\right\|_{0}^{2} \leq \frac{1}{2} k_{0},
$$

for all $1 \leq m \leq N$.

Moreover, we derive from (2.8) that

$$
\begin{aligned}
\left(d_{t t} u_{\epsilon}^{n}, v\right) & +a_{\epsilon}\left(d_{t} u_{\epsilon}^{n}, v\right)+b\left(d_{t} u_{\epsilon}^{n}, u_{\epsilon}^{n}, v\right)+b\left(u_{\epsilon}^{n-1}, d_{t} u_{\epsilon}^{n}, v\right) \\
& =\left(\frac{1}{\Delta t} \int_{t_{n-1}}^{t_{n}} f_{t}(t) d t, v\right), \forall v \in X .
\end{aligned}
$$

By taking $v=2 d_{t} u_{\epsilon}^{n} \Delta t$ in (3.11) and using (2.5) and (3.3), we get

$$
\begin{aligned}
\left\|d_{t} u_{\epsilon}^{n}\right\|_{0}^{2}-\left\|d_{t} u_{\epsilon}^{n-1}\right\|_{0}^{2} & +2 \nu\left\|A_{\epsilon}^{1 / 2} d_{t} u_{\epsilon}^{n}\right\|_{0}^{2} \Delta t+2 b\left(d_{t} u_{\epsilon}^{n}, u_{\epsilon}^{n}, d_{t} u_{\epsilon}^{n}\right) \Delta t \\
& \leq 2\left(\int_{t_{n-1}}^{t_{n}} f_{t}(t) d t, d_{t} u_{\epsilon}^{n}\right) .
\end{aligned}
$$


Due to (2.1) and (2.4), we have

$$
\begin{aligned}
2\left|b\left(d_{t} u_{\epsilon}^{n}, u_{\epsilon}^{n}, d_{t} u_{\epsilon}^{n}\right)\right| & \leq c\left\|d_{t} u_{\epsilon}^{n}\right\|_{0}\left\|A^{1 / 2} d_{t} u_{\epsilon}^{n}\right\|_{0}\left\|A^{1 / 2} u_{\epsilon}^{n}\right\|_{0} \\
& +c\left\|d_{t} u_{\epsilon}^{n}\right\|_{0}^{1 / 2}\left\|A^{1 / 2} d_{t} u_{\epsilon}^{n}\right\|_{0}^{3 / 2}\left\|A^{1 / 2} u_{\epsilon}^{n}\right\|_{0}^{1 / 2}\left\|u_{\epsilon}^{n}\right\|_{0}^{1 / 2} \\
& \leq \frac{\nu}{2}\left\|A_{\epsilon}^{1 / 2} d_{t} u_{\epsilon}^{n}\right\|_{0}^{2}+d_{n}\left\|d_{t} u_{\epsilon}^{n}\right\|_{0}^{2}, \\
\left|\left(\int_{t_{n-1}}^{t_{n}} f_{t}(t) d t, d_{t} u_{\epsilon}^{n}\right)\right| & \leq \frac{\nu}{2}\left\|A_{\epsilon}^{1 / 2} d_{t} u_{\epsilon}^{n}\right\|_{0}^{2} \Delta t+\nu^{-1} c \int_{t_{n-1}}^{t_{n}}\left\|f_{t}\right\|_{0}^{2} d t .
\end{aligned}
$$

Combining these inequalities with (3.12) yields

$$
\begin{aligned}
\left\|d_{t} u_{\epsilon}^{n}\right\|_{0}^{2} & -\left\|d_{t} u_{\epsilon}^{n-1}\right\|_{0}^{2}+\nu\left\|A_{\epsilon}^{1 / 2} d_{t} u_{\epsilon}^{n}\right\|_{0}^{2} \Delta t \\
& \leq d_{n}\left\|d_{t} u_{\epsilon}^{n}\right\|_{0}^{2} \Delta t+\nu^{-1} c \int_{t_{n-1}}^{t_{n}}\left\|f_{t}(t)\right\|_{0}^{2} d t .
\end{aligned}
$$

Summing (3.13) from 1 to $m$ and using (2.9), (3.9)-(3.10), we get

$$
\left\|d_{t} u_{\epsilon}^{m}\right\|_{0}^{2}+\nu \Delta t \sum_{n=1}^{m}\left\|A_{\epsilon}^{1 / 2} d_{t} u_{\epsilon}^{n}\right\|_{0}^{2} \leq \kappa, \forall 1 \leq m \leq N .
$$

Finally, we derive from (2.8), (3.5) and the inf-sup condition [7] that

$$
\begin{aligned}
\nu\left\|A_{\epsilon} u_{\epsilon}^{n}\right\|_{0} & \leq\left\|d_{t} u_{\epsilon}^{n}\right\|_{0}+\left\|B\left(u_{\epsilon}^{n}, u_{\epsilon}^{n}\right)\right\|_{0}+\left\|f\left(t_{n}\right)\right\|_{0} \\
\left\|p_{\epsilon}^{n}\right\|_{1} & \leq c\left\|d_{t} u_{\epsilon}^{n}\right\|_{0}+\nu c\left\|A u_{\epsilon}^{n}\right\|_{0}+c\left\|B\left(u_{\epsilon}^{n}, u_{\epsilon}^{n}\right)\right\|_{0}+c\left\|f\left(t_{n}\right)\right\|_{0}
\end{aligned}
$$

Using (2.3)-(2.4), we have

$$
\begin{aligned}
\left\|B\left(u_{\epsilon}^{n}, u_{\epsilon}^{n}\right)\right\|_{0} & \leq c\left\|A^{1 / 2} u_{\epsilon}^{n}\right\|_{0}\left\|u_{\epsilon}^{n}\right\|_{L^{\infty}} \leq c\left\|A^{1 / 2} u_{\epsilon}^{n}\right\|_{0}\left\|A u_{\epsilon}^{n}\right\|_{0}^{1 / 2}\left\|u_{\epsilon}^{n}\right\|_{0}^{1 / 2} \\
& \leq \frac{\nu}{2}\left\|A_{\epsilon} u_{\epsilon}^{n}\right\|_{0}+\nu^{-1} c\left\|u_{\epsilon}^{n}\right\|_{0}\left\|A^{1 / 2} u_{\epsilon}^{n}\right\|_{0}^{2} .
\end{aligned}
$$

Combining these inequalities with (3.15) and (3.16) and using (2.4), we get

$$
\begin{aligned}
\nu\left\|A_{\epsilon} u_{\epsilon}^{n}\right\|_{0}^{2} & \leq \nu^{-1}\left\|d_{t} u_{\epsilon}^{n}\right\|_{0}^{2}+\nu^{-3} c\left\|u_{\epsilon}^{n}\right\|_{0}^{2}\left\|A_{\epsilon}^{1 / 2} u_{\epsilon}^{n}\right\|_{0}^{4}+\nu^{-1} c\left\|f\left(t_{n}\right)\right\|_{0}^{2} \\
\left\|p_{\epsilon}^{n}\right\|_{1}^{2} & \leq c\left(\left\|d_{t} u_{\epsilon}^{n}\right\|_{0}^{2}+\nu^{2}\left\|A_{\epsilon} u_{\epsilon}^{n}\right\|_{0}^{2}\right)+c \nu^{-2}\left\|u_{\epsilon}^{n}\right\|_{0}^{2}\left\|A_{\epsilon}^{1 / 2} u_{\epsilon}^{n}\right\|_{0}^{4}+\left\|f\left(t_{n}\right)\right\|_{0}^{2}, \\
\Delta t \sum_{n=1}^{m}\left\|p_{\epsilon}^{n}\right\|_{1}^{2} & \leq c \Delta t \sum_{n=1}^{m}\left(\left\|d_{t} u_{\epsilon}^{n}\right\|_{0}^{2}+\nu^{2}\left\|A_{\epsilon} u_{\epsilon}^{n}\right\|_{0}^{2}\right) \\
& +c \Delta t \sum_{n=1}^{m}\left(\nu^{-2}\left\|u_{\epsilon}^{n}\right\|_{0}^{2}\left\|A_{\epsilon}^{1 / 2} u_{\epsilon}^{n}\right\|_{0}^{4}+\left\|f\left(t_{n}\right)\right\|_{0}^{2}\right) .
\end{aligned}
$$

Combining (3.17)-(3.19) with (3.9)-(3.10) and (3.14), we completed the proof of Theorem 3.1.

Theorem 3.2. Under the assumptions of Theorem [3.1, it holds that

$$
\tau\left(t_{m}\right)\left\|A_{\epsilon}^{1 / 2} d_{t} u_{\epsilon}^{m}\right\|_{0}^{2}+\Delta t \sum_{n=1}^{m} \tau\left(t_{n}\right)\left(\left\|d_{t t} u_{\epsilon}^{n}\right\|_{0}^{2}+\left\|A_{\epsilon} d_{t} u_{\epsilon}^{n}\right\|_{0}^{2}+\left\|d_{t} p_{\epsilon}^{n}\right\|_{1}^{2}\right) \leq \kappa,
$$

for all $1 \leq m \leq N$. 
Proof. Taking $v=2 \nu^{-1} d_{t t} u_{\epsilon}^{n} \Delta t$ in (3.11) yields

$$
2\left(A_{\epsilon}^{1 / 2} d_{t} u_{\epsilon}^{n}, A_{\epsilon}^{1 / 2}\left(d_{t} u_{\epsilon}^{n}-d_{t} u_{\epsilon}^{n-1}\right)\right)+2 \nu^{-1}\left\|d_{t t} u_{\epsilon}^{n}\right\|_{0}^{2} \Delta t+2 \nu^{-1} b\left(d_{t} u_{\epsilon}^{n}, u_{\epsilon}^{n}, d_{t t} u_{\epsilon}^{n}\right) \Delta t
$$

$$
+2 \nu^{-1} b\left(u_{\epsilon}^{n}, d_{t} u_{\epsilon}^{n}, d_{t t} u_{\epsilon}^{n}\right) \Delta t=2 \nu^{-1}\left(\int_{t_{n-1}}^{t_{n}} f_{t}(t) d t, d_{t t} u_{\epsilon}^{n}\right) .
$$

Due to (2.1)-(2.4), we have

$$
\begin{aligned}
2 \nu^{-1}\left|b\left(d_{t} u_{\epsilon}^{n}, u_{\epsilon}^{n}, d_{t t} u_{\epsilon}^{n}\right)\right| & +2 \nu^{-1}\left|b\left(d_{t} u_{\epsilon}^{n}, u_{\epsilon}^{n}, d_{t t} u_{\epsilon}^{n}\right)\right| \\
& \leq c \nu^{-1}\left\|A^{1 / 2} d_{t} u_{\epsilon}^{n}\right\|_{0}\left\|A u_{\epsilon}^{n}\right\|_{0}\left\|d_{t t} u_{\epsilon}^{n}\right\|_{0} \\
& \leq \frac{1}{4 \nu}\left\|d_{t t} u_{\epsilon}^{n}\right\|_{0}^{2}+\nu^{-1} c\left\|A_{\epsilon} u_{\epsilon}^{n}\right\|_{0}^{2}\left\|A_{\epsilon}^{1 / 2} d_{t} u_{\epsilon}^{n}\right\|_{0}^{2}, \\
2 \nu^{-1}\left|\left(\int_{t_{n-1}}^{t_{n}} f_{t}(t) d t, d_{t} u_{\epsilon}^{n}\right)\right| & \leq \frac{1}{4 \nu}\left\|d_{t t} u_{\epsilon}^{n}\right\|_{0}^{2} \Delta t+\nu^{-1} c \int_{t_{n-1}}^{t_{n}}\left\|f_{t}\right\|_{0}^{2} d t .
\end{aligned}
$$

Combining these inequalities with (3.21) yields

$$
\begin{aligned}
2 \tau\left(t_{n}\right)\left(A_{\epsilon}^{1 / 2} d_{t} u_{\epsilon}^{n}, A_{\epsilon}^{1 / 2}\left(d_{t} u_{\epsilon}^{n}\right.\right. & \left.\left.-d_{t} u_{\epsilon}^{n-1}\right)\right)+\nu^{-1} \tau\left(t_{n}\right)\left\|d_{t t} u_{\epsilon}^{n}\right\|_{0}^{2} \Delta t \\
& \leq \nu^{-1} c\left\|A_{\epsilon} u_{\epsilon}^{n}\right\|_{0}^{2}\left\|A_{\epsilon}^{1 / 2} d_{t} u_{\epsilon}^{n}\right\|_{0}^{2}+\nu^{-1} c \int_{t_{n-1}}^{t_{n}}\left\|f_{t}(t)\right\|_{0}^{2} d t .
\end{aligned}
$$

Summing (3.22) from 1 to $m$ and noting

$$
\begin{aligned}
2 \tau\left(t_{n}\right) & \left(A_{\epsilon}^{1 / 2} d_{t} u_{\epsilon}^{n}, A_{\epsilon}^{1 / 2}\left(d_{t} u_{\epsilon}^{n}-d_{t} u_{\epsilon}^{n-1}\right)\right) \\
\geq & \tau\left(t_{n}\right)\left\|A_{\epsilon}^{1 / 2} d_{t} u_{\epsilon}^{n}\right\|_{0}^{2}-\tau\left(t_{n-1}\right)\left\|A_{\epsilon}^{1 / 2} d_{t} u_{\epsilon}^{n-1}\right\|_{0}^{2} \\
& -\mid A_{\epsilon}^{1 / 2} d_{t} u_{\epsilon}^{n-1} \|_{0}^{2} \Delta t, 2 \leq n \leq N, \\
2 \tau\left(t_{1}\right) & \left(A_{\epsilon}^{1 / 2} d_{t} u_{\epsilon}^{1}, A_{\epsilon}^{1 / 2}\left(d_{t} u_{\epsilon}^{1}-d_{t} u_{\epsilon}^{0}\right)\right) \\
\geq & \tau\left(t_{1}\right)\left\|A_{\epsilon}^{1 / 2} d_{t} u_{\epsilon}^{1}\right\|_{0}^{2}-\left\|A_{\epsilon} u_{\epsilon}^{1}-A u_{0}\right\|_{0}\left\|d_{t} u_{\epsilon}^{0}\right\|_{0},
\end{aligned}
$$

we obtain

$$
\begin{aligned}
\tau\left(t_{m}\right)\left\|A_{\epsilon}^{1 / 2} d_{t} u_{\epsilon}^{m}\right\|_{0}^{2} & +\nu^{-1} \Delta t \sum_{n=1}^{m} \tau\left(t_{n}\right)\left\|d_{t t} u_{\epsilon}^{n}\right\|_{0}^{2} \\
& \leq\left\|A_{\epsilon} u_{\epsilon}^{1}-A u_{0}\right\|_{0}\left\|d_{t} u_{\epsilon}^{0}\right\|_{0}+\Delta t \sum_{n=1}^{m}\left\|A_{\epsilon}^{1 / 2} d_{t} u_{\epsilon}^{n}\right\|_{0}^{2} \\
& +\nu^{-1} c \Delta t \sum_{n=1}^{m}\left\|A_{\epsilon} u_{\epsilon}^{n}\right\|_{0}^{2}\left\|A_{\epsilon}^{1 / 2} d_{t} u_{\epsilon}^{n}\right\|_{0}^{2}+\nu^{-1} c \int_{0}^{T}\left\|f_{t}(t)\right\|_{0}^{2} d t .
\end{aligned}
$$

Combining (3.23) with (3.2) yields

$$
\tau\left(t_{m}\right)\left\|A_{\epsilon}^{1 / 2} d_{t} u_{\epsilon}^{m}\right\|_{0}^{2} \quad+\quad \nu^{-1} \Delta t \sum_{n=1}^{m} \tau\left(t_{n}\right)\left\|d_{t t} u_{\epsilon}^{n}\right\|_{0}^{2} \leq \kappa, \forall 1 \leq m \leq N .
$$

Finally, we derive from (2.8) and the inf-sup condition [7] that

$$
\begin{aligned}
\nu\left\|A_{\epsilon} d_{t} u_{\epsilon}^{n}\right\|_{0} \leq & \left\|d_{t t} u_{\epsilon}^{n}\right\|_{0}+\left\|B\left(d_{t} u_{\epsilon}^{n}, u_{\epsilon}^{n}\right)\right\|_{0}+\left\|B\left(u_{\epsilon}^{n}, d_{t} u_{\epsilon}^{n}\right)\right\|_{0}+\left\|f_{t}\left(t_{n}\right)\right\|_{0} \\
\left\|d_{t} p_{\epsilon}^{n}\right\|_{1} \leq & c\left\|d_{t t} u_{\epsilon}^{n}\right\|_{0}+\nu c\left\|A d_{t} u_{\epsilon}^{n}\right\|_{0}+c\left\|B\left(d_{t} u_{\epsilon}^{n}, u_{\epsilon}^{n}\right)\right\|_{0} \\
& +c\left\|B\left(u_{\epsilon}^{n}, d_{t} u_{\epsilon}^{n}\right)\right\|_{0}+c\left\|f\left(t_{n}\right)\right\|_{0} .
\end{aligned}
$$


Using (2.1)-(2.4), we have

$$
\left\|B\left(d_{t} u_{\epsilon}^{n}, u_{\epsilon}^{n}\right)\right\|_{0}+\left\|B\left(d_{t} u_{\epsilon}^{n}, u_{\epsilon}^{n}\right)\right\|_{0} \leq c\left\|A_{\epsilon} u_{\epsilon}^{n}\right\|_{0}\left\|A_{\epsilon}^{1 / 2} d_{t} u_{\epsilon}^{n}\right\|_{0} .
$$

Combining this inequality with (3.25) and (3.26), we get

$$
\begin{aligned}
\nu^{2} \Delta t \sum_{n=1}^{m} \tau\left(t_{n}\right)\left\|A_{\epsilon} d_{t} u_{\epsilon}^{n}\right\|_{0}^{2} & \leq c \Delta t \sum_{n=1}^{m} \tau\left(t_{n}\right)\left\|d_{t t} u_{\epsilon}^{n}\right\|_{0}^{2} \\
& +c \Delta t \sum_{n=1}^{m}\left\|A_{\epsilon} u_{\epsilon}^{n}\right\|_{0}^{2}\left\|A_{\epsilon}^{1 / 2} d_{t} u_{\epsilon}^{n}\right\|_{0}^{2}+c \Delta t \sum_{n=1}^{m}\left\|f_{t}\left(t_{n}\right)\right\|_{0}^{2}, \\
(3.27) \quad & \leq \Delta t \sum_{n=1}^{m} \tau\left(t_{n}\right)\left(\left\|d_{t t} u_{\epsilon}^{n}\right\|_{0}^{2}+\nu^{2}\left\|A_{\epsilon} d_{t} u_{\epsilon}^{n}\right\|_{0}^{2}\right) \\
\Delta t \sum_{n=1}^{m} \tau\left(t_{n}\right)\left\|d_{t} p_{\epsilon}^{n}\right\|_{1}^{2} & \leq c \Delta t \sum_{n=1}^{m}\left(\left\|A_{\epsilon} u_{\epsilon}^{n}\right\|_{0}^{2}\left\|A_{\epsilon}^{1 / 2} d_{t} u_{\epsilon}^{n}\right\|_{0}^{2}+\left\|f_{t}\left(t_{n}\right)\right\|_{0}^{2}\right),
\end{aligned}
$$

Combining (3.27)-(3.28) with (3.24) and using (3.2), we have completed the proof of Theorem 3.2

\section{Finite element penalty method of the Navier-Stokes equations}

Let $h>0$ be a real positive parameter. The finite element space pair $\left(X_{h}, M_{h}\right)$ of $(X, M)$ is characterized by $J_{h}=J_{h}(\Omega)$, a partitioning of $\bar{\Omega}$ into triangles $K$, assumed to be uniformly regular as $h \rightarrow 0$. For further details, the reader can refer to Ciarlet [6] and Girault and Raviart [7].

Let $\rho_{h}: M \rightarrow M_{h}$ denote the $L^{2}$-orthogonal projections defined by

$$
\left(\rho_{h} q, q_{h}\right)=\left(q, q_{h}\right), \forall q \in M, q_{h} \in M_{h} .
$$

For the finite element space pair $\left(X_{h}, M_{h}\right)$, we will make the following assumption. $\left(A_{2}\right)$ There exists a mapping $r_{h}: D(A) \cap X \rightarrow X_{h}$ such that

$$
\begin{aligned}
& \left(\operatorname{div}\left(u-r_{h} u\right), q_{h}\right)=0, \forall q_{h} \in M_{h}, \\
& \left\|A^{1 / 2}\left(u-r_{h} u\right)\right\|_{0} \leq c h\|A u\|_{0},\left\|p-\rho_{h} p\right\|_{0} \leq c h\|p\|_{1}, \forall p \in H^{1}(\Omega) \cap M,
\end{aligned}
$$

and the inverse inequality

$$
\left\|A^{1 / 2} v_{h}\right\|_{0} \leq c h^{-1}\left\|v_{h}\right\|_{0}, \forall v_{h} \in X_{h},
$$

holds as well as the discrete inf-sup condition

$$
\left\|q_{h}\right\|_{0} \leq c \sup _{v_{h} \in X_{h}} \frac{\left(\operatorname{div} v_{h}, q_{h}\right)}{\left\|A^{1 / 2} v_{h}\right\|_{0}}, \forall q_{h} \in M_{h} .
$$

Example 4.1 (Girault-Raviart []). If we set

$$
\begin{aligned}
X_{h} & =\left\{v_{h} \in C^{0}(\Omega)^{2} \cap X ;\left.v_{h}\right|_{K} \in P_{2}(K)^{2}, \forall K \in J_{h}(\Omega)\right\}, \\
M_{h} & =\left\{q_{h} \in M ;\left.q_{h}\right|_{K} \in P_{0}(K), \forall K \in J_{h}(\Omega)\right\},
\end{aligned}
$$

then $\left(X_{h}, M_{h}\right)$ satisfies $\left(A_{2}\right)$. 
Now, we consider the finite element discretization of (2.8). We define $\left\{u_{\epsilon h}^{n}\right\}_{n=1}^{N} \subset$ $X_{h}$ and $\left\{p_{\epsilon h}^{n}\right\}_{n=1}^{N} \subset M_{h}$ as the finite element approximations of $\left\{u_{\epsilon}^{n}\right\}_{n=1}^{N} \subset X$ and $\left\{p_{\epsilon}^{n}\right\}_{n=1}^{N} \subset M$, which satisfy the recursive linear equation

$$
\begin{aligned}
\left(d_{t} u_{\epsilon h}^{n}, v_{h}\right) & +a\left(u_{\epsilon h}^{n}, v_{h}\right)-d\left(v_{h}, p_{\epsilon h}^{n}\right)+d\left(u_{\epsilon h}^{n}, q_{h}\right)+\frac{\epsilon}{\nu}\left(p_{\epsilon h}^{n}, q_{h}\right)+b\left(u_{\epsilon h}^{n}, u_{\epsilon h}^{n}, v_{h}\right) \\
(4.5) & =\left(f\left(t_{n}\right), v_{h}\right), \forall\left(v_{h}, q_{h}\right) \in\left(X_{h}, M_{h}\right),
\end{aligned}
$$

where $u_{\epsilon h}^{0}=r_{h} u_{0}, p_{\epsilon h}^{0}=0$.

Theorem 4.1. Assume that $\left(A_{1}\right),\left(A_{2}\right)$ and $\epsilon c_{0} \leq 1, \Delta t \kappa_{0} \leq 1$ are valid. Then it holds that

$$
\begin{aligned}
\left\|u_{\epsilon h}^{m}\right\|_{0}^{2} & +\Delta t \sum_{n=1}^{m}\left\|A^{1 / 2} u_{\epsilon h}^{n}\right\|_{0}^{2} \leq \kappa \\
\left\|A^{1 / 2} u_{\epsilon h}^{m}\right\|_{0}^{2} & +\Delta t \sum_{n=1}^{m}\left\|d_{t} u_{\epsilon h}^{n}\right\|_{0}^{2} \leq \kappa+\kappa h^{-2} \Delta t \sum_{n=1}^{m}\left\|A^{1 / 2}\left(u_{\epsilon}^{n}-u_{\epsilon h}^{n}\right)\right\|_{0}^{2}\left\|u_{\epsilon h}^{n}\right\|_{0}^{2} \\
& +\kappa h^{-2} \Delta t \sum_{n=1}^{m}\left\|u_{\epsilon}^{n}-u_{\epsilon h}^{n}\right\|_{0}^{2}\left\|A^{1 / 2} u_{\epsilon h}^{n}\right\|_{0}^{2},
\end{aligned}
$$

for all $1 \leq m \leq N$.

Proof. The proof of (4.6) is exactly similar to that of (3.4) in the proof of Theorem 3.1. It can be omitted. Moreover, we can obtain from (4.5) that

$$
\begin{aligned}
\left(d_{t} u_{\epsilon h}^{n}, v_{h}\right) & +a\left(u_{\epsilon h}^{n}, v_{h}\right)-d\left(v_{h}, p_{\epsilon h}^{n}\right) \\
& +d\left(d_{t} u_{\epsilon h}^{n}, q_{h}\right)+\frac{\epsilon}{\nu}\left(d_{t} p_{\epsilon h}^{n}, q_{h}\right)+b\left(u_{\epsilon h}^{n}, u_{\epsilon h}^{n}, v_{h}\right) \\
= & \left(f\left(t_{n}\right), v_{h}\right), \forall\left(v_{h}, q_{h}\right) \in\left(X_{h}, M_{h}\right) .
\end{aligned}
$$

Taking $\left(v_{h}, q_{h}\right)=2\left(d_{t} u_{\epsilon h}^{n}, p_{\epsilon h}^{n}\right) \Delta t$ in (4.8) and using (3.6), we obtain

$$
\begin{aligned}
2\left\|d_{t} u_{\epsilon h}^{n}\right\|_{0}^{2} \Delta t & +\nu\left\|A^{1 / 2} u_{\epsilon h}^{n}\right\|_{0}^{2}-\nu\left\|A^{1 / 2} u_{\epsilon h}^{n-1}\right\|_{0}^{2}+\frac{\epsilon}{\nu}\left(\left\|p_{\epsilon h}^{n}\right\|_{0}^{2}-\left\|p_{\epsilon h}^{n-1}\right\|_{0}^{2}\right) \\
& +2 b\left(u_{\epsilon h}^{n}, u_{\epsilon h}^{n}, d_{t} u_{\epsilon h}^{n}\right) \Delta t=2\left(f\left(t_{n}\right), d_{t} u_{\epsilon h}^{n}\right) \Delta t .
\end{aligned}
$$

By using (2.1)-(2.3), (2.5) and (4.3), we have

$$
\begin{aligned}
2\left|b\left(u_{\epsilon h}^{n}, u_{\epsilon h}^{n}, d_{t} u_{\epsilon h}^{n}\right)\right| & \leq 2\left|b\left(u_{\epsilon h}^{n}-u_{\epsilon}^{n}, u_{\epsilon h}^{n}, d_{t} u_{\epsilon h}^{n}\right)\right|+2\left|b\left(u_{\epsilon}^{n}, u_{\epsilon h}^{n}, d_{t} u_{\epsilon h}^{n}\right)\right|, \\
2\left|b\left(u_{\epsilon}^{n}, u_{\epsilon h}^{n}, d_{t} u_{\epsilon h}^{n}\right)\right| & \leq c\left\|A u_{\epsilon}^{n}\right\|_{0}\left\|A^{1 / 2} u_{\epsilon h}^{n}\right\|\left\|_{0}\right\| d_{t} u_{\epsilon h}^{n} \|_{0} \\
& \leq \frac{1}{4}\left\|d_{t} u_{\epsilon h}^{n}\right\|_{0}^{2}+c\left\|A u_{\epsilon}^{n}\right\|_{0}^{2}\left\|A^{1 / 2} u_{\epsilon h}^{n}\right\|_{0}^{2} \\
2\left|b\left(u_{\epsilon h}^{n}-u_{\epsilon}^{n}, u_{\epsilon h}^{n}, d_{t} u_{\epsilon h}^{n}\right)\right| & \leq c\left\|u_{\epsilon h}^{n}-u_{\epsilon}^{n}\right\|_{L^{4}}\left\|u_{\epsilon h}^{n}\right\|_{L^{4}}\left\|A^{1 / 2} d_{t} u_{\epsilon h}^{n}\right\|_{0} \\
& +c\left\|A^{1 / 2}\left(u_{\epsilon h}^{n}-u_{\epsilon}^{n}\right)\right\|_{0}^{2}\left\|u_{\epsilon h}^{n}\right\|_{L^{4}}\left\|A^{1 / 2} d_{t} u_{\epsilon h}^{n}\right\|_{L^{4}} \\
& \leq \frac{1}{4}\left\|d_{t} u_{\epsilon h}^{n}\right\|_{0}^{2}+c h^{-2}\left\|A^{1 / 2}\left(u_{\epsilon}^{n}-u_{\epsilon h}^{n}\right)\right\|_{0}^{2}\left\|u_{\epsilon h}^{n}\right\|_{0}^{2} \\
& +c h^{-2}\left\|u_{\epsilon}^{n}-u_{\epsilon h}^{n}\right\|_{0}^{2}\left\|A^{1 / 2} u_{\epsilon h}^{n}\right\|_{0}^{2}, \\
2\left|\left(f\left(t_{n}\right), d_{t} u_{\epsilon}^{n}\right)\right| & \leq \frac{1}{4}\left\|d_{t} u_{\epsilon h}^{n}\right\|_{0}^{2}+c\left\|f\left(t_{n}\right)\right\|_{0}^{2} .
\end{aligned}
$$


Combining these estimates with (4.9) yields

$$
\begin{aligned}
\nu\left\|A^{1 / 2} u_{\epsilon h}^{n}\right\|_{0}^{2} & -\nu\left\|A^{1 / 2} u_{\epsilon h}^{n-1}\right\|_{0}^{2}+\left\|d_{t} u_{\epsilon h}^{n}\right\|_{0}^{2} \Delta t+\frac{\epsilon}{\nu}\left(\left\|p_{\epsilon h}^{n}\right\|_{0}^{2}-\left\|p_{\epsilon h}^{n-1}\right\|_{0}^{2}\right) \\
& \leq c\left\|A u_{\epsilon}^{n}\right\|_{0}^{2}\left\|A^{1 / 2} u_{\epsilon h}^{n}\right\|_{0}^{2} \Delta t+c h^{-2}\left\|A^{1 / 2}\left(u_{\epsilon}^{n}-u_{\epsilon h}^{n}\right)\right\|_{0}^{2}\left\|u_{\epsilon h}^{n}\right\|_{0}^{2} \Delta t \\
& +\left\|u_{\epsilon}^{n}-u_{\epsilon h}^{n}\right\|_{0}^{2}\left\|A^{1 / 2} u_{\epsilon h}^{n}\right\|_{0}^{2} \Delta t+c\left\|f\left(t_{n}\right)\right\|_{0}^{2} \Delta t .
\end{aligned}
$$

Summing this inequality from 1 to $m$ and using Theorem 3.1 and (2.4), we obtain (4.7).

In order to derive the error estimates of the finite element penalty method, we also need the Galerkin projection $R_{h}:(X, M) \rightarrow X_{h}, Q_{h}:(X, M) \rightarrow M_{h}$ defined by

$$
\begin{aligned}
a\left(u-R_{h}(u, p), v_{h}\right) & -d\left(v_{h}, p-Q_{h}(u, p)\right)+d\left(u-R_{h}(u, p), q_{h}\right) \\
& +\frac{\epsilon}{\nu}\left(p-Q_{h}(u, p), q_{h}\right)=0, \forall\left(v_{h}, q_{h}\right) \in\left(X_{h}, M_{h}\right),
\end{aligned}
$$

for all $(u, p) \in(X, M)$ with $\operatorname{div} u+\frac{\epsilon}{\nu} p=0$.

By using a similar argument to that used by Layton and Tobiska in [15], the following approximate properties can be proved.

Lemma 4.2. Under the assumptions of Theorem 4.1, the Galerkin projection $\left(R_{h}, Q_{h}\right)$ satisfies

$$
\begin{aligned}
\left\|u-R_{h}(u, p)\right\|_{0} & +h\left\|A^{1 / 2}\left(u-R_{h}(u, p)\right)\right\|_{0}+h\left\|p-Q_{h}(u, p)\right\|_{0} \\
& \leq c_{\nu} h\left(\left\|A^{1 / 2} u\right\|_{0}+\|p\|_{0}\right), \forall(u, p) \in(X, M),
\end{aligned}
$$

with divu $+\frac{\epsilon}{\nu} p=0$, and

$$
\begin{aligned}
\left\|u-R_{h}(u, p)\right\|_{0} & +h\left\|A^{1 / 2}\left(u-R_{h}(u, p)\right)\right\|_{0}+h\left\|p-Q_{h}(u, p)\right\|_{0} \\
& \leq c_{\nu} h^{2}\left(\|A u\|_{0}+\|p\|_{1}\right), \forall(u, p) \in\left(D(A), H^{1}(\Omega) \cap M\right),
\end{aligned}
$$

with divu $+\frac{\epsilon}{\nu} p=0$, where $c_{\nu}$ is a general positive constant depending only on $\Omega$ and $\nu$, which may stand for different values at its different occurrences.

Proof. The stability of the Galerkin projection follows simply by (4.4) and (4.11), namely

$$
\begin{aligned}
\nu\left\|A^{1 / 2} R_{h}(u, p)\right\|_{0}^{2} & \leq \frac{\nu}{2}\left\|A^{1 / 2} R_{h}(u, p)\right\|_{0}^{2}+c_{v}\left(\left\|A^{1 / 2} u\right\|_{0}^{2}+\|p\|_{0}^{2}\right), \\
\left\|Q_{h}(u, p)\right\|_{0} & \leq c_{\nu}\left(\left\|A^{1 / 2} R_{h}(u, p)\right\|_{0}+\left\|A^{1 / 2} u\right\|_{0}+\|p\|_{0}\right) .
\end{aligned}
$$

Now (4.14)-(4.15) and the triangle inequality give

$$
\left\|A^{1 / 2}\left(u-R_{h}(u, p)\right)\right\|_{0}+\left\|p-Q_{h}(u, p)\right\|_{0} \leq c_{\nu}\left(\left\|A^{1 / 2} u\right\|_{0}+\|p\|_{0}\right),
$$

for all $(u, p) \in(X, M)$ with $\operatorname{div} u+\frac{\epsilon}{\nu} p=0$.

Next, we introduce the dual problem: find $(\Phi, \Psi) \in(X, M)$ such that for all $(v, q) \in(X, M)$

$$
a(v, \Phi)+d(v, \Psi)-d(\Phi, q)+\frac{\epsilon}{\nu}(q, \Psi)=\left(v, u-R_{h}(u, p)\right) .
$$

In view of Lemma [2.1, we can prove that problem (4.17) admits a unique solution $(\Phi, \Psi)$ satisfying

$$
\|A \Phi\|_{0}+\|\Psi\|_{1} \leq c_{\nu}\left\|u-R_{h}(u, p)\right\|_{0} .
$$


Now, setting $v=u-R_{h}(u, p), q=p-Q_{h}(u, p)$ in (4.17) and $\left(v_{h}, q_{h}\right)=\left(r_{h} \Phi, \rho_{h} \Psi\right)$ in (4.11) and using (4.2) and (4.18), we find

$$
\begin{aligned}
\left|u-R_{h}(u, p)\right|^{2} & =a\left(u-R_{h}(u, p), \Phi-r_{h} \Phi\right)+d\left(u-R_{h}(u, p), \Psi-\rho_{h} \Psi\right) \\
& -d\left(\Phi-r_{h} \Phi, p-Q_{h}(u, p)\right)+\frac{\epsilon}{\nu}\left(p-Q_{h}(u, p), \Psi-\rho_{h} \Psi\right) \\
(4.19) \leq & c_{\nu} h\left(\left\|A^{1 / 2}\left(u-R_{h}(u, p)\right)\right\|_{0}+\left\|p-Q_{h}(u, p)\right\|_{0}\right)\left(\|A \Phi\|_{0}+\|\Psi\|_{1}\right) \\
& \leq c_{\nu} h\left(\left\|A^{1 / 2}\left(u-R_{h}(u, p)\right)\right\|_{0}+\left\|p-Q_{h}(u, p)\right\|_{0}\right)\left\|u-R_{h}(u, p)\right\|_{0} .
\end{aligned}
$$

Combining (4.19) with (4.16) yields (4.12).

Let $(u, p) \in\left(D(A), H^{1}(\Omega) \cap M\right)$ with $\operatorname{div} u+\frac{\epsilon}{\nu} p=0$. Then, we derive from (4.1) - (4.2) and (4.11) that

$$
\begin{aligned}
\| A^{1 / 2}\left(r_{h} u-R_{h}(u, p)\right) & \left\|_{0}+\right\| \rho_{h} p-Q_{h}(u, p) \|_{0} \\
& \leq c_{\nu}\left(\left\|A^{1 / 2}\left(u-r_{h} u\right)\right\|_{0}+\left\|\rho_{h} p-p\right\|_{0}\right) \\
& \leq c_{\nu} h\left(\|A u\|_{0}+\|p\|_{1}\right) .
\end{aligned}
$$

Thus the triangles inequality and (4.2) give

$$
\left\|A^{1 / 2}\left(u-R_{h}(u, p)\right)\right\|_{0}+\left\|p-Q_{h}(u, p)\right\|_{0} \leq c_{\nu} h\left(\|A u\|_{0}+\|p\|_{1}\right) .
$$

It now follows from (4.20) and (4.19) that

$$
\left\|u-R_{h}(u, p)\right\|_{0} \leq c_{\nu} h^{2}\left(\|A u\|_{0}+\|p\|_{1}\right) .
$$

Thus, (4.20) and (4.21) imply 4.13).

\section{Optimal ERROR ANALYSIS}

In this section, our aim is to estimate some bounds for the error $\left(u_{\epsilon}^{n}-u_{\epsilon h}^{n}, p_{\epsilon}^{n}-p_{\epsilon h}^{n}\right)$ and then to obtain the optimal bound of the error $\left(u\left(t_{n}\right)-u_{\epsilon h}^{n}, p\left(t_{n}\right)-p_{\epsilon h}^{n}\right)$.

Lemma 5.1. Under the assumptions of Theorem 4.1, the error estimate

$$
\left\|u_{\epsilon}^{m}-u_{\epsilon h}^{m}\right\|_{0}^{2}+\Delta t \sum_{n=1}^{m}\left\|A^{1 / 2}\left(u_{\epsilon}^{n}-u_{\epsilon h}^{n}\right)\right\|_{0}^{2} \leq \kappa h^{2}
$$

holds for all $1 \leq m \leq N$.

Proof. Subtracting (4.5) from (2.8) with $(v, q)=\left(v_{h}, q_{h}\right)$ and using (4.1), we obtain

$$
\begin{aligned}
((I- & \left.\left.r_{h}\right) d_{t} u_{\epsilon}^{n}+d_{t} e^{n}, v_{h}\right)+a\left(\left(I-r_{h}\right) u_{\epsilon}^{n}+e^{n}, v_{h}\right) \\
& -d\left(v_{h},\left(I-\rho_{h}\right) p_{\epsilon}^{n}+\eta^{n}\right)+d\left(e^{n}, q_{h}\right) \\
& +b\left(\left(I-r_{h}\right) u_{\epsilon}^{n}+e^{n}, u_{\epsilon}^{n}, v_{h}\right)+b\left(u_{\epsilon h}^{n},\left(I-r_{h}\right) u_{\epsilon}^{n}+e^{n}, v_{h}\right) \\
& +\frac{\epsilon}{\nu}\left(\left(I-\rho_{h}\right) p_{\epsilon}^{n}+\eta^{n}, q_{h}\right)=0, \quad \forall\left(v_{h}, q_{h}\right) \in\left(X_{h}, M_{h}\right),
\end{aligned}
$$

where $\left(e^{n}, \eta^{n}\right)=\left(r_{h} u_{\epsilon}^{n}-u_{\epsilon h}^{n}, \rho_{h} p_{\epsilon}^{n}-p_{\epsilon h}^{n}\right)$. Taking $\left(v_{h}, q_{h}\right)=2\left(e^{n}, \eta^{n}\right) \Delta t$ in (5.2) and using (2.5) and (3.3), we get

$$
\begin{aligned}
\left\|e^{n}\right\|_{0}^{2} & -\left\|e^{n-1}\right\|_{0}^{2}+2 \nu\left\|A^{1 / 2} e^{n}\right\|_{0}^{2} \Delta t+\frac{\epsilon}{\nu}\left\|\eta^{n}\right\|_{0}^{2} \Delta t+2 b\left(e^{n}, u_{\epsilon}^{n}, e^{n}\right) \Delta t \\
& +2 b\left(\left(I-r_{h}\right) u_{\epsilon}^{n}, u_{\epsilon}^{n}, e^{n}\right) \Delta t+2 b\left(u_{\epsilon h}^{n},\left(I-r_{h}\right) u_{\epsilon}^{n}, e^{n}\right) \Delta t \\
\leq & \frac{\epsilon}{\nu}\left\|\left(I-\rho_{h}\right) p_{\epsilon}^{n}\right\|_{0}^{2} \Delta t+2 d\left(e^{n},\left(I-\rho_{h}\right) p_{\epsilon}^{n}\right) \Delta t \\
& -2 a\left(\left(I-r_{h}\right) u_{\epsilon}^{n}, e^{n}\right) \Delta t-2\left(\left(I-r_{h}\right) d_{t} u_{\epsilon}^{n}, e^{n}\right) \Delta t .
\end{aligned}
$$


Using (2.1) and (4.2), we have

$$
\begin{aligned}
2\left|b\left(e^{n}, u_{\epsilon}^{n}, e^{n}\right)\right| & \leq c\left\|e^{n}\right\|_{L^{4}}^{2}\left\|A^{1 / 2} u_{\epsilon}^{n}\right\|_{0}+c\left\|A^{1 / 2} e^{n}\right\|_{0}\left\|u_{\epsilon}^{n}\right\|_{L^{4}}\left\|e^{n}\right\|_{L^{4}} \\
& \leq \frac{\nu}{4}\left\|A^{1 / 2} e^{n}\right\|_{0}^{2}+d_{n}\left\|e^{n}\right\|_{0}^{2}, \\
2\left|b\left(\left(I-r_{h}\right) u_{\epsilon}^{n}, u_{\epsilon}^{n}, e^{n}\right)\right| & +2\left|b\left(u_{\epsilon h}^{n},\left(I-r_{h}\right) u_{\epsilon}^{n}, e^{n}\right)\right| \\
& \leq c\left\|A^{1 / 2}\left(I-r_{h}\right) u_{\epsilon}^{n}\right\|_{0}\left(\left\|A^{1 / 2} u_{\epsilon}^{n}\right\|_{0}+\left\|A^{1 / 2} u_{\epsilon h}^{n}\right\|_{0}\right)\left\|A^{1 / 2} e^{n}\right\|_{0} \\
& \leq \frac{\nu}{4}\left\|A^{1 / 2} e^{n}\right\|_{0}^{2}+c_{\nu} h^{2}\left\|A u_{\epsilon}^{n}\right\|_{0}^{2}\left(\left\|A^{1 / 2} u_{\epsilon}^{n}\right\|_{0}^{2}+\left\|A^{1 / 2} u_{\epsilon h}^{n}\right\|_{0}^{2}\right), \\
\frac{\epsilon}{\nu}\left\|\left(I-\rho_{h}\right) p_{\epsilon}^{n}\right\|_{0}^{2} & +2\left|d\left(e^{n},\left(I-\rho_{h}\right) p_{\epsilon}^{n}\right)\right| \leq \frac{\nu}{4}\left\|A^{1 / 2} e^{n}\right\|_{0}^{2}+c_{\nu} h^{2}\left\|p_{\epsilon}^{n}\right\|_{1}^{2}, \\
2\left|a\left(\left(I-r_{h}\right) u_{\epsilon}^{n}, e^{n}\right)\right| & \leq \frac{\nu}{8}\left\|A^{1 / 2} e^{n}\right\|_{0}^{2}+c_{\nu} h^{2}\left\|A u_{\epsilon}^{n}\right\|_{0}^{2}, \\
2\left|\left(\left(I-r_{h}\right) d_{t} u_{\epsilon}^{n}, e^{n}\right)\right| & \leq \frac{\nu}{8}\left\|A^{1 / 2} e^{n}\right\|_{0}^{2}+c_{\nu} h^{2}\left\|A^{1 / 2} d_{t} u_{\epsilon}^{n}\right\|_{0}^{2} .
\end{aligned}
$$

Combining these estimates with (5.3) yields

$$
\begin{aligned}
\left\|e^{n}\right\|_{0}^{2} & -\left\|e^{n-1}\right\|_{0}^{2}+\nu\left\|A^{1 / 2} e^{n}\right\|_{0}^{2} \Delta t \leq d_{n}\left\|e^{n}\right\|_{0}^{2} \Delta t \\
& +c_{\nu} h^{2}\left(\left\|A^{1 / 2} u_{\epsilon}^{n}\right\|_{0}^{2}+\left\|A^{1 / 2} u_{\epsilon h}^{n}\right\|_{0}^{2}\right)\left\|A u_{\epsilon}^{n}\right\|_{0}^{2} \Delta t \\
& +c_{\nu} h^{2}\left(\left\|A u_{\epsilon}^{n}\right\|_{0}^{2}+\left\|A^{1 / 2} d_{t} u_{\epsilon}^{n}\right\|_{0}^{2}+\left\|p_{\epsilon}^{n}\right\|_{1}^{2}\right) \Delta t, \quad \forall 1 \leq n \leq N .
\end{aligned}
$$

Summing (5.4) from 1 to $m$ and using Theorem 3.1. Theorem 4.1 and (2.4), we obtain

$$
\left\|e^{m}\right\|_{0}^{2}+\nu \Delta t \sum_{n=1}^{m}\left\|A^{1 / 2} e^{n}\right\|_{0}^{2} \leq \Delta t \sum_{n=1}^{m} d_{n}\left\|e^{n}\right\|_{0}^{2}+\kappa h^{2} .
$$

Due to (3.10), we can apply Lemma 2.3 to (5.5) and obtain

$$
\left\|e^{m}\right\|_{0}^{2}+\nu \Delta t \sum_{n=1}^{m}\left\|A^{1 / 2} e^{n}\right\|_{0}^{2} \leq \kappa h^{2} .
$$

Moreover, by using (2.4), (4.2) and Theorem 3.1 we have

$$
\begin{aligned}
& \left\|\left(I-r_{h}\right) u_{\epsilon}^{m}\right\|_{0}^{2}+\nu \Delta t \sum_{n=1}^{m}\left\|A^{1 / 2}\left(I-r_{h}\right) u_{\epsilon}^{n}\right\|_{0}^{2} \leq c h^{2}\left(\left\|A^{1 / 2} u_{\epsilon}^{n}\right\|^{2}+\nu \Delta t \sum_{n=1}^{m}\left\|A u_{\epsilon}^{n}\right\|_{0}^{2}\right) \\
& \quad \leq \kappa h^{2} .
\end{aligned}
$$

Combining (5.6) with (5.7) has completed the proof of Lemma 5.1

Lemma 5.2. Under the assumptions of Theorem 4.1, we have

$$
\left\|A^{1 / 2}\left(u_{\epsilon}^{m}-u_{\epsilon h}^{m}\right)\right\|_{0}^{2}+\Delta t \sum_{n=1}^{m}\left\|p_{\epsilon}^{n}-p_{\epsilon h}^{n}\right\|_{0}^{2} \leq \kappa h^{2}, \forall 1 \leq m \leq N .
$$

Proof. Subtracting (4.5) from (2.8) with $(v, q)=\left(v_{h}, q_{h}\right)$ and setting $e^{n}=R_{h}^{n}-$ $u_{\epsilon h}^{n}, \eta^{n}=Q_{h}^{n}-p_{\epsilon h}^{n}, R_{h}^{n}=R_{h}\left(u_{\epsilon}^{n}, p_{\epsilon}^{n}\right), Q_{h}^{n}=Q_{h}\left(u_{\epsilon}^{n}, p_{\epsilon}^{n}\right)$, we deduce

$$
\begin{aligned}
\left(d_{t} e^{n}, v_{h}\right) & +a\left(e^{n}, v_{h}\right)-d\left(v_{h}, \eta^{n}\right)+d\left(d_{t} e^{n}, q_{h}\right)+\frac{\epsilon}{\nu}\left(d_{t} \eta^{n}, q_{h}\right) \\
& +b\left(u_{\epsilon}^{n}-R_{h}^{n}+e^{n}, u_{\epsilon}^{n}, v_{h}\right)+b\left(u_{\epsilon}^{n}, u_{\epsilon}^{n}-R_{h}^{n}+e^{n}, v_{h}\right) \\
& -b\left(u_{\epsilon}^{n}-u_{\epsilon h}^{n}, u_{\epsilon}^{n}-u_{\epsilon h}^{n}, v_{h}\right)=-\left(d_{t} u_{\epsilon}^{n}-R_{h}\left(d_{t} u_{\epsilon}^{n}, d_{t} p_{\epsilon}^{n}\right), v_{h}\right),
\end{aligned}
$$


for all $\left(v_{h}, q_{h}\right) \in\left(X_{h}, M_{h}\right)$ with $1 \leq n \leq N$. Taking $\left(v_{h}, q_{h}\right)=2\left(d_{t} e^{n}, \eta^{n}\right) \Delta t$ in (5.9), we get

$$
\begin{aligned}
& 2\left\|d_{t} e^{n}\right\|_{0}^{2} \Delta t+\nu\left(\left\|A^{1 / 2} e^{n}\right\|_{0}^{2}-\left\|A^{1 / 2} e^{n-1}\right\|_{0}^{2}\right)+\frac{\epsilon}{\nu}\left(\left\|\eta^{n}\right\|_{0}^{2}-\left\|\eta^{n-1}\right\|_{0}^{2}\right) \\
&+2 b\left(u_{\epsilon}^{n}-R_{h}^{n}+e^{n}, \bar{u}_{h}^{n}, d_{t} e^{n}\right) \Delta t+2 b\left(u_{\epsilon h}^{n}, u_{\epsilon}^{n}-R_{h}^{n}+e^{n}, d_{t} e^{n}\right) \Delta t \\
&(5.10) \quad-2 b\left(u_{\epsilon}^{n}-u_{\epsilon h}^{n}, u_{\epsilon}^{n}-u_{\epsilon h}^{n}, v_{h}\right) \Delta t=-2\left(d_{t} u_{\epsilon}^{n}-R_{h}\left(d_{t} u_{\epsilon}^{n}, d_{t} p_{\epsilon}^{n}\right), d_{t} e^{n}\right) \Delta t,
\end{aligned}
$$

Due to $\left(u_{\epsilon}^{0}, p_{\epsilon}^{0}\right)=\left(u_{0}, 0\right)$, we derive from (4.2) and (4.9) that

$$
\begin{aligned}
\left\|A^{1 / 2}\left(u_{\epsilon}^{0}-R_{h}\left(u_{\epsilon}^{0}, p_{\epsilon}^{0}\right)\right)\right\|_{0} & +\left\|p_{\epsilon}^{0}-Q_{h}\left(u_{\epsilon}^{0}, p_{\epsilon}^{0}\right)\right\|_{0} \leq c_{\nu} h\left\|A u_{0}\right\|_{0} \\
\left\|A^{1 / 2} e^{0}\right\|_{0}+\left\|\eta^{0}\right\|_{0} \leq & \left\|A^{1 / 2}\left(u_{0}-r_{h} u^{0}\right)\right\|_{0}+\left\|A^{1 / 2}\left(u_{\epsilon}^{0}-R_{h}\left(u_{\epsilon}^{0}, p_{\epsilon}^{0}\right)\right)\right\|_{0} \\
& +\left\|Q_{h}\left(u_{\epsilon}^{0}, p_{\epsilon}^{0}\right)\right\|_{0} \leq c_{\nu} h\left\|A u_{0}\right\|_{0} .
\end{aligned}
$$

Using again (2.1)-(2.3) and (4.3), it follows that

$$
\begin{aligned}
& 2 \mid b\left(e^{n}, u_{\epsilon}^{n}, d_{t} e^{n}\right)|+2| b\left(u_{\epsilon}^{n}, e^{n}, d_{t} e^{n}\right) \mid \leq c\left\|A u_{\epsilon}^{n}\right\|_{0}\left\|A^{1 / 2} e^{n}\right\|_{0}\left\|d_{t} e^{n}\right\|_{0} \\
& \leq \frac{1}{4}\left\|d_{t} e^{n}\right\|_{0}^{2}+c\left\|A u_{\epsilon}^{n}\right\|_{0}^{2}\left\|A^{1 / 2} e^{n}\right\|_{0}^{2}, \\
& 2\left|b\left(u_{\epsilon}^{n}-R_{h}^{n}, u_{\epsilon}^{n}, d_{t} e^{n}\right)\right|+\left|b\left(u_{\epsilon}^{n}, u_{\epsilon}^{n}-R_{h}^{n}, d_{t} e^{n}\right)\right| \\
& \leq c\left\|A u_{\epsilon}^{n}\right\|_{0}\left\|A^{1 / 2}\left(u_{\epsilon}^{n}-R_{h}^{n}\right)\right\|_{0}\left\|d_{t} e^{n}\right\|_{0} \\
& \leq \frac{1}{4}\left\|d_{t} e^{n}\right\|_{0}^{2}+c\left\|A u_{\epsilon}^{n}\right\|_{0}^{2}\left\|A^{1 / 2}\left(u_{\epsilon}^{n}-R_{\epsilon}^{n}\right)\right\|_{0}^{2}, \\
& 2\left|b\left(u_{\epsilon}^{n}-u_{\epsilon h}^{n}, u_{\epsilon}^{n}-u_{\epsilon h}^{n}, d_{t} e^{n}\right)\right| \leq c\left(h^{-1}\left\|A^{1 / 2}\left(u_{\epsilon}^{n}-u_{\epsilon h}^{n}\right)\right\|_{0}\left\|u_{\epsilon}^{n}-u_{\epsilon h}^{n}\right\|_{0}\right. \\
&\left.+\left\|A^{1 / 2}\left(u_{\epsilon}^{n}-u_{\epsilon h}^{n}\right)\right\|_{0}^{2}\right)\left\|d_{t} e^{n}\right\|_{0} \\
& \leq \frac{1}{4}\left\|d_{t} e^{n}\right\|_{0}^{2}+c\left(h^{-2}\left\|A^{1 / 2}\left(u_{\epsilon}^{n}-u_{\epsilon h}^{n}\right)\right\|_{0}^{2}\left\|u_{\epsilon}^{n}-u_{\epsilon h}^{n}\right\|_{0}^{2}\right. \\
&\left.+\left\|A^{1 / 2}\left(u_{\epsilon}^{n}-u_{\epsilon h}^{n}\right)\right\|_{0}^{4}\right), \\
& 2\left|\left(d_{t}\left(u-R_{h}(u, p)\right), d_{t} e^{n}\right)\right| \leq \frac{1}{4}\left\|d_{t} e^{n}\right\|_{0}^{2}+\left\|d_{t} u_{\epsilon}^{n}-R_{h}\left(d_{t} u_{\epsilon}^{n}, d_{t} p_{\epsilon}^{n}\right)\right\|_{0}^{2} .
\end{aligned}
$$

Combining these inequalities with (5.10) and using Lemma 4.2 results in

$$
\begin{aligned}
& \nu\left\|A^{1 / 2} e^{n}\right\|_{0}^{2}-\nu\left\|A^{1 / 2} e^{n-1}\right\|_{0}^{2}+\left\|d_{t} e^{n}\right\|_{0}^{2} \Delta t+\frac{\epsilon}{\nu}\left(\left\|\eta^{n}\right\|_{0}^{2}-\left\|\eta^{n-1}\right\|_{0}^{2}\right) \\
& \leq c\left\|A u_{\epsilon}^{n}\right\|_{0}^{2}\left(\left\|A^{1 / 2} e^{n}\right\|_{0}^{2}+h^{2}\left\|A u_{\epsilon}^{n}\right\|_{0}^{2}+h^{2}\left\|p_{\epsilon}^{n}\right\|_{1}^{2}\right) \Delta t \\
&+c h^{4}\left(\left\|A d_{t} u_{\epsilon}^{n}\right\|_{0}^{2}+\left\|d_{t} p_{\epsilon}^{n}\right\|_{1}^{2}\right) \Delta t \\
&+c\left(h^{-2}\left\|A^{1 / 2}\left(u_{\epsilon}^{n}-u_{\epsilon h}^{n}\right)\right\|_{0}^{2}\left\|u_{\epsilon}^{n}-u_{\epsilon h}^{n}\right\|_{0}^{2}+\left\|A^{1 / 2}\left(u_{\epsilon}^{n}-u_{\epsilon h}^{n}\right)\right\|_{0}^{4}\right) \Delta t .
\end{aligned}
$$

From Theorems 3.1 and 4.1, Lemma 5.1 and the fact that $h^{2} \leq c_{1} \Delta t \leq c_{1} \tau\left(t_{n}\right)$, we derive from (5.13) that

$$
\begin{aligned}
& \nu\left\|A^{1 / 2} e^{n}\right\|_{0}^{2}-\nu\left\|A^{1 / 2} e^{n-1}\right\|_{0}^{2}+\left\|d_{t} e^{n}\right\|_{0}^{2} \Delta t+\frac{\epsilon}{\nu}\left(\left\|\eta^{n}\right\|_{0}^{2}-\left\|\eta^{n-1}\right\|_{0}^{2}\right) \\
& \leq \kappa\left(\left\|A^{1 / 2} e^{n}\right\|_{0}^{2}+h^{2}\left\|A u_{\epsilon}^{n}\right\|_{0}^{2}+h^{2}\left\|p_{\epsilon}^{n}\right\|_{1}^{2}\right) \Delta t \\
&+c h^{2} \tau\left(t_{n}\right)\left(\left\|A d_{t} u_{\epsilon}^{n}\right\|_{0}^{2}+\left\|d_{t} p_{\epsilon}^{n}\right\|_{1}^{2}\right) \Delta t \\
&+\kappa\left(\left\|A^{1 / 2}\left(u_{\epsilon}^{n}-u_{\epsilon h}^{n}\right)\right\|_{0}^{2}+\left\|A^{1 / 2}\left(u_{\epsilon}^{n}-u_{\epsilon h}^{n}\right)\right\|_{0}^{2}\right) \Delta t .
\end{aligned}
$$


Summing (5.14) from 1 to $m$ and using Theorems [3.1] and [3.2 Lemma [5.1] and (5.12) leads to

$$
\nu\left\|A^{1 / 2} e^{m}\right\|_{0}^{2}+\Delta t \sum_{n=1}^{m}\left\|d_{t} e^{n}\right\|_{0}^{2} \leq \kappa h^{2}, \forall 1 \leq m \leq N .
$$

Moreover, by using Lemma 4.2. Theorems 3.1 and 3.2 and the fact that $h^{2} \leq$ $c_{1} \tau\left(t_{n}\right)$, we have

$$
\begin{aligned}
\nu \| A^{1 / 2}\left(u_{\epsilon}^{n}\right. & \left.-R_{h}\left(u_{\epsilon}^{n}, p_{\epsilon}^{n}\right)\right)\left\|_{0}^{2}+\Delta t \sum_{n=1}^{m}\right\| d_{t} u_{\epsilon}^{n}-R_{h}\left(d_{t} u_{\epsilon}^{n}, d_{t} p_{\epsilon}^{n}\right) \|_{0}^{2} \\
& \leq c_{\nu} h^{2}\left(\left\|A u_{\epsilon}^{n}\right\|_{0}^{2}+\left\|p_{\epsilon}^{n}\right\|_{1}^{2}\right)+c_{\nu} h^{2} \Delta t \sum_{n=1}^{m} \tau\left(t_{n}\right)\left(\left\|A d_{t} u_{\epsilon}^{n}\right\|_{0}^{2}+\left\|d_{t} p_{\epsilon}^{n}\right\|_{1}^{2}\right) \\
(5.16) \leq \kappa h^{2}, \forall 1 \leq m \leq N . & \leq
\end{aligned}
$$

Combining (5.15) with (5.16) yields

$$
\left\|A^{1 / 2}\left(u_{\epsilon}^{m}-u_{\epsilon h}^{m}\right)\right\|_{0}^{2}+\Delta t \sum_{n=1}^{m}\left\|d_{t} u_{\epsilon}^{n}-d_{t} u_{\epsilon h}^{n}\right\|_{0}^{2} \leq \kappa h^{2}, \quad \forall 1 \leq m \leq N .
$$

Finally, by using (5.2), (4.4) and (2.1), we obtain

$$
\begin{aligned}
\left\|\rho_{h} p_{\epsilon}^{n}-p_{\epsilon h}^{n}\right\|_{0} & \leq c\left\|d_{t} u_{\epsilon}^{n}-d_{t} u_{\epsilon h}^{n}\right\|_{0}+c \nu\left\|A^{1 / 2}\left(u_{\epsilon}^{n}-u_{\epsilon h}^{n}\right)\right\|_{0} \\
& +c\left\|A^{1 / 2}\left(u_{\epsilon}^{n}-u_{\epsilon h}^{n}\right)\right\|_{0}\left(\left\|A^{1 / 2} u_{\epsilon}^{n}\right\|_{0}+\left\|A^{1 / 2} u_{\epsilon h}^{n}\right\|_{0}\right) \\
& +c\left\|\left(I-\rho_{h}\right) p_{\epsilon}^{n}\right\|_{0} .
\end{aligned}
$$

Using Theorem 3.1. Theorem 4.1 and Lemma 4.2, we derive from (5.18) that

$$
\begin{aligned}
\left\|p_{\epsilon}^{n}-p_{\epsilon h}^{n}\right\|_{0}^{2} \Delta t & \leq c\left\|d_{t} u_{\epsilon}^{n}-d_{t} u_{\epsilon h}^{n}\right\|_{0}^{2} \Delta t+\kappa\left\|A^{1 / 2}\left(u_{\epsilon}^{n}-u_{\epsilon h}^{n}\right)\right\|_{0}^{2} \Delta t \\
& +c\left\|\left(I-\rho_{h}\right) p_{\epsilon}^{n}\right\|_{0}^{2} \Delta t .
\end{aligned}
$$

Summing (5.19) from 1 to $m$ and using (5.17), (4.2) and Theorem 3.1 we find

$$
\Delta t \sum_{n=1}^{m}\left\|p_{\epsilon}^{n}-p_{\epsilon h}^{n}\right\|_{0}^{2} \leq \kappa h^{2}, \forall 1 \leq m \leq N .
$$

Combining (5.20) with (5.17) yields (5.8).

By combining Lemma 5.2 with Theorem 2.2 we obtain the optimal error estimate result.

Theorem 5.3. Under the assumptions of Theorem 4.1, the optimal error estimate

$$
\begin{aligned}
\tau^{2}\left(t_{m}\right)\left\|A^{1 / 2}\left(u\left(t_{m}\right)-u_{\epsilon h}^{m}\right)\right\|_{0}^{2} & +\Delta t \sum_{n=1}^{m} \tau^{2}\left(t_{n}\right)\left\|p\left(t_{n}\right)-p_{\epsilon h}^{n}\right\|_{0}^{2} \\
& \leq \kappa\left(\epsilon^{2}+\Delta t^{2}+h^{2}\right), \quad \forall 1 \leq m \leq N
\end{aligned}
$$

holds.

\section{ACKNOWLEDGMENTS}

The author wishes to thank the referees for their valuable comments and suggestions which helped to improve the results of this paper. 


\section{REFERENCES}

[1] M. Bercovier, Perturbation of mixed variational problems, applications to mixed finite element methods, RAIRO Anal. Numer., 12(1978), pp. 221-236. MR0509973 (80b:49031)

[2] B. Brefort, Attractor for the penalty Navier-Stokes equations, SIAM J. Math. Anal., 19(1988), pp. 1-21. MR0924541 (89b:35135)

[3] F. Brezzi And J. Pitkaranta, On the stabilization of finite element approximation of the Stokes problem, in Efficient Solutions of Elliptic Systems, Notes on Numerical Fluid Mechanics, Vol. 10, W. Hackbusch. ed., Vieweg, 1984, pp. 11-19. MF0804083 (86j:65147)

[4] A. J. Chorin, Numerical solution of the Navier-Stokes equations, Math. Comp., 22(1968), pp. 745-762. MR0242392 (39:3723)

[5] A. J. Chorin, On the convergence of discrete approximations to the Navier-Stokes equations, Math. Comp., 23(1969), pp. 341-353. MR0242393(39:3724)

[6] P. G. Ciarlet, The Finite Element Method for Elliptic Problems, North-Holland, Amsterdam, 1978. MR0520174 (58:25001)

[7] V. Girault and P. A. Raviart, Finite Element Method for Navier-Stokes Equations: theory and algorithms, Springer-Verlag, Berlin, Heidelberg, 1987. MR0851383 (88b:65129)

[8] Yinnian He, Fully Discrete Stabilized Finite Element Method for the Time-Dependent Navier-Stokes Equations, IMA J. Numer. Anal., 23(2003), pp. 1-23. MR2011345 (2004m:65151)

[9] Yinnian He, Yanping Lin and Weiwei Sun, Stabilized finite element method for the NavierStokes problem, submitted.

[10] J. G. Heywood And R. Rannacher, Finite element approximation of the nonstationary Navier-Stokes problem I: Regularity of solutions and second-order error estimates for spatial discretization, SIAM J. Numer. Anal., 19(1982), pp. 275-311. MR0650052 (83d:65260)

[11] J. G. HeYwood And R. RANnACher, Finite element approximation of the nonstationary Navier-Stokes problem IV: Error analysis for second-order time discretization, SIAM J. Numer. Anal., 27(1990), pp. 353-384. MR1043610 (92c:65133)

[12] Aixiang Huang and Kaitai Li, Penalty method for the nonstationary Navier-Stokes equations, Acta Mathematicae Applicatae Sinca (in Chinese), 17(1994), pp. 473-480. MR 1333935 (96b:65092)

[13] T. J. R. Hughes, W. T. Liu, And A. J. Brooks, Finite element analysis of incompressible viscous flows by the penalty function formulation, J. Comp. Phys, 30(1979), pp. 1-60. MR:0524162 (80b:76008)

[14] N. KechKar AND D. Silvester, Analysis of locally stabilized mixed finite element methods for the Stokes problem, Math. Comp., 58(1992), pp. 1-10. MR.1106973 (92e:65138)

[15] W. Layton and L. Tobiska, A two-level method with backtraking for the Navier-Stokes equations, SIAM J. Numer. Anal., 35(1998), pp. 2035-2056. MR.1639994 (99g:65115)

[16] J. SHEn, On error estimates of the penalty method for unsteady Navier-Stokes equations, SIAM J. Numer. Anal., 32(1995), pp. 386-403. MR1324294 (96d:65153)

[17] J. SHEn, On error estimates of some higher order projection and penalty-projection methods for Navier-Stokes equations, Numer. Math. 62(1992), pp. 49-73. MR1159045|(93a:35122)

[18] J. SHEn, On error estimates of the projection method for Navier-Stokes equations, SIAM J. Numer. Anal., 29(1992), pp. 57-77. MR1149084 (92m:35213)

[19] R. Temam, Une méthode d'approximation des solutions des équations de Navier-Stokes, Bull. Soc. Math. France, 98(1968), pp.115-152. MR0237972 (38:6249)

[20] R. Temam, Sur méthode d'approximation de la solution des équations de Navier-Stokes par la méthode des pas fractionnaires I, Arch. Rational Mech. Anal., 32(1969), pp. 135-153. MR 0237973(38:6250)

[21] R. Temam, Sur méthode d'approximation de la solution des équations de Navier-Stokes par la méthode des pas fractionnaires II, Arch. Rational Mech. Anal., 33(1969), pp. 377-385. MR 0244654(39:5968)

Faculty of Science, Xi'an Jiaotong University, Xi'an 710049, People's Republic of CHINA

E-mail address: heyn@mail.xjtu.edu.cn 